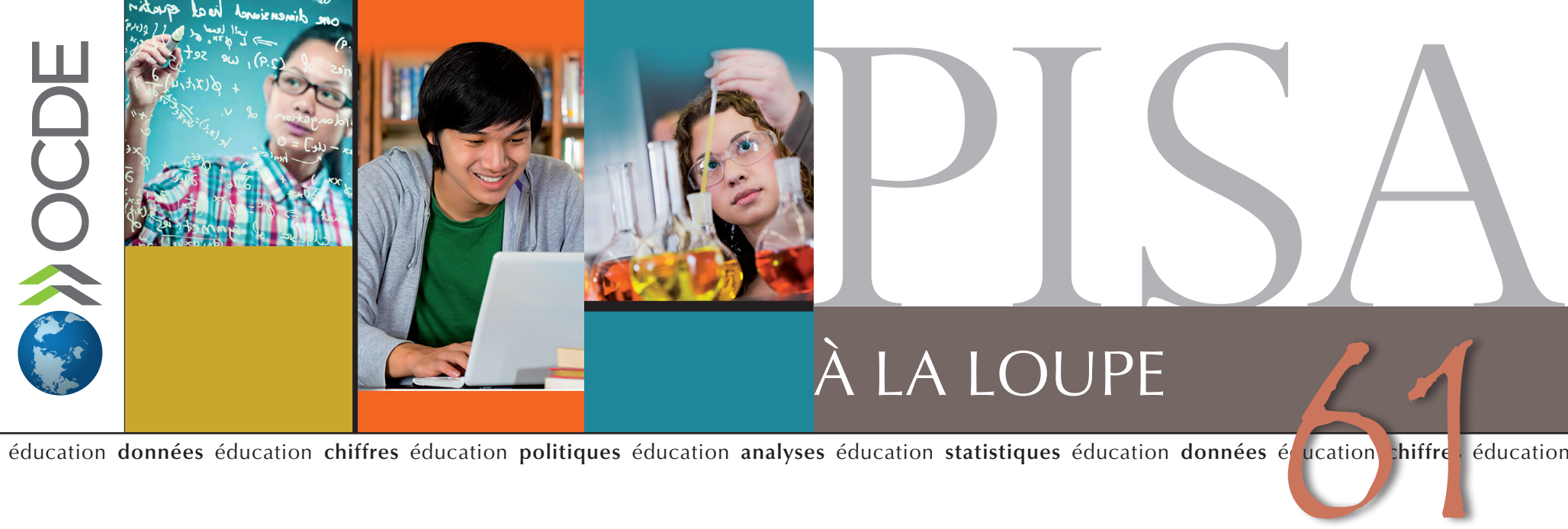

\title{
La mémorisation : une stratégie payante pour l'apprentissage des mathématiques?
}

- Les élèves de 15 ans des pays d'Asie de l'Est sont moins nombreux à indiquer avoir recours à la mémorisation que ceux de certains pays anglophones auxquels on les compare souvent.

- Les garçons n'indiquent utiliser davantage les stratégies de mémorisation que les filles pour apprendre les mathématiques dans aucun système d'éducation ayant participé à l'enquête PISA.

- La mémorisation peut fonctionner comme stratégie d’apprentissage pour les problèmes faciles, mais est peu susceptible d'être efficace si elle est utilisée seule pour résoudre des problèmes complexes de mathématiques.

L'aire d'un cercle est égale au carré de son rayon multiplié par $\pi$. Le carré de l'hypoténuse est égal à la somme des carrés des deux autres côtés. Ces formules vous rappellent quelque chose ? Vous souvenez-vous comment vous les avez apprises? En comprenant leur signification et en les mettant en application dans tout un éventail de problèmes de mathématiques s'inscrivant dans une multiplicité de contextes différents? Ou en les apprenant par cœur et en les mettant en application dans une série de problèmes de mathématiques se ressemblant tous les uns les autres ? Tôt ou tard, la méthode finit par compter. Les élèves ne faisant pas l'effort de comprendre les concepts mathématiques peuvent certes réussir dans certains contextes scolaires, mais l'absence d'un raisonnement approfondi, critique et créatif peut gravement les pénaliser plus tard, lorsqu'ils seront confrontés à des problèmes de la vie réelle sortant de l'ordinaire. Rien de surprenant alors à ce que de nombreux systèmes d'éducation de par le monde s'interrogent sur le rôle que les stratégies de mémorisation, telles que l'apprentissage par cœur et les exercices de répétition, doivent jouer dans le processus d'apprentissage. Prenez par exemple la polémique actuelle en Angleterre sur la question des tables de multiplication : les uns y affirment que les faire mémoriser aux enfants avant l'âge de 9 ans, comme le préconise le programme scolaire national, revient à faire un pas dans la mauvaise direction, tandis que les autres continuent de penser que c'est un moyen d'aider les enfants à acquérir les fondamentaux.

Qui sont les élèves ayant le plus recours à la mémorisation?

La réponse en surprendra peut-être plus d'un.

L'enquête PISA souhaitait comprendre comment les élèves apprennent les mathématiques. Un questionnaire distribué aux élèves participants leur demandait ainsi d'indiquer, parmi un choix de trois options, quelle stratégie d'apprentissage décrivait le mieux leur approche des mathématiques. Parmi les options proposées, l'une correspondait toujours à une stratégie de mémorisation, telle que « apprendre par cœur ", "vérifier que les points déjà travaillés sont bien retenus » ou " revoir les exemples encore et encore ». Un indice de mémorisation a ensuite été dérivé du nombre de fois où l'élève avait choisi une stratégie de mémorisation. Les valeurs de cet indice sont comprises entre 0 , lorsque l'élève a systématiquement choisi une autre stratégie d'apprentissage, telle que « faire le lien entre différents concepts » et " déterminer exactement quels sont les points les plus importants à apprendre », et 4, lorsque l'élève a systématiquement choisi l'option correspondant à une stratégie de mémorisation. 


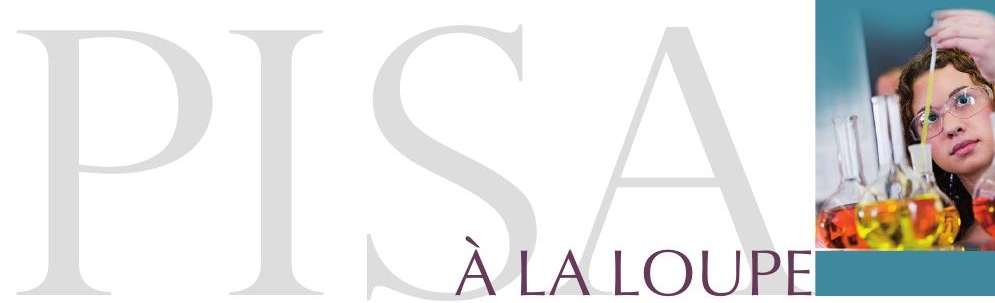

Quels liens entre les caractéristiques des élèves et les pratiques des enseignants, et l'utilisation de stratégies de mémorisation par les élèves ?

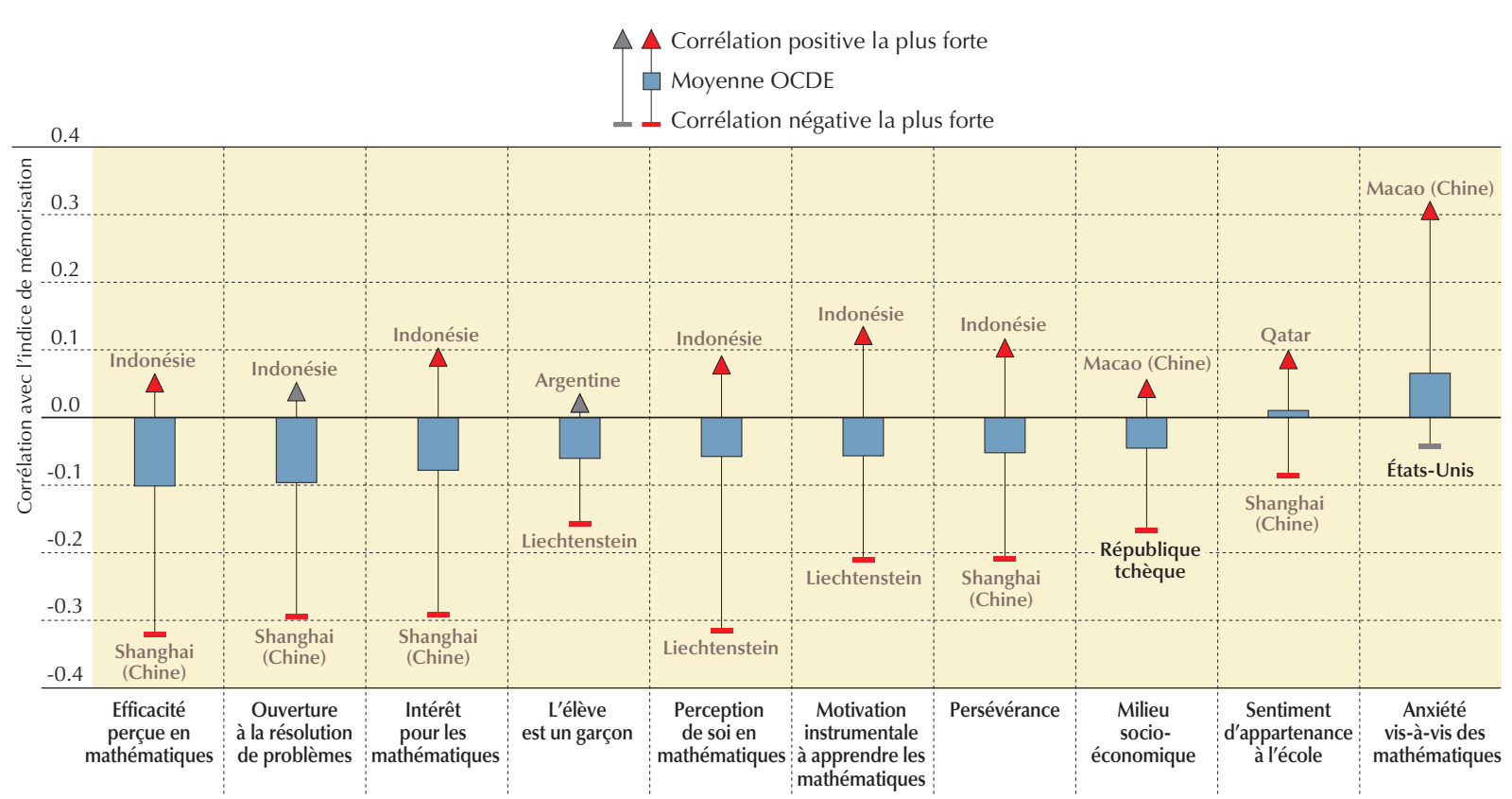

Remarques : les coefficients de corrélation statistiquement significatifs sont indiqués en rouge. Tous les coefficients de corrélation pour la moyenne OCDE sont statistiquement significatifs.

Source : OCDE, Base de données PISA 2012

Les élèves utilisant principalement les stratégies de mémorisation peuvent obtenir de bons résultats aux questions faciles...

La mémorisation est souvent perçue comme une stratégie élémentaire mieux adaptée à la résolution de problèmes de mathématiques familiers ne nécessitant qu'une compréhension peu approfondie des concepts mathématiques. "HIT-PARADE, question 1 ", un item rendu public de l'évaluation PISA 2012, constitue un bon exemple de ce type de problèmes. Il s'agit d'une question à choix multiple basée sur un diagramme simple en bâtons.

Quelque $87 \%$ des élèves des 48 systèmes d'éducation ayant administré cette question y ont répondu correctement. Il s'agit là de l'item le plus facile parmi ceux rendus publics. Les élèves indiquant utiliser des stratégies de mémorisation pour apprendre les mathématiques ont obtenu à cet item un taux de réussite assez semblable à celui des élèves indiquant utiliser d'autres stratégies d'apprentissage. Dans certains systèmes d'éducation, comme en Albanie, en Lituanie et en Slovénie, les élèves indiquant utiliser des stratégies de mémorisation étaient même plus susceptibles de répondre correctement à ce problème.

\section{... mais les problèmes complexes nécessitent plus qu'une bonne mémoire.}

Les résultats sont très différents pour l'item " PORTE À TAMBOUR, question 2 », la question de mathématiques la plus difficile de l'évaluation PISA 2012. Cette dernière appelle en effet une réponse construite ou ouverte face à un problème nécessitant un niveau poussé de raisonnement géométrique et de créativité, impliquant l'enchaînement de plusieurs étapes, et dépendant dans une large mesure de la capacité des élèves à traduire une situation réelle en problème mathématique. Seuls $3 \%$ des élèves participants y ont répondu correctement.

Une analyse des résultats de l'enquête PISA et des réponses des élèves au questionnaire montre que dans les pays de l'OCDE, chaque augmentation d'une unité de l'indice de mémorisation entraîne une diminution de près de $31 \%$ de la probabilité de répondre correctement à cette question. Les élèves indiquant utiliser le plus des stratégies de mémorisation lorsqu'ils étudient sont quatre fois moins susceptibles de réussir à résoudre ce problème que ceux indiquant les utiliser le moins. 
